\title{
Evaluierung der Wirksamkeit und Verträglichkeit von $5 \%$ iger Kaliumhydroxid-Lösung in der Behandlung von Molluscum contagiosum im Kindesalter
}

\author{
Evaluation of the Efficacy and Tolerability of $5 \%$ Potassium Hydroxide Solution in the Treatment \\ of Molluscum Contagiosum in Childhood
}

Autoren

Institute

\section{T. Jansen ', R. Romiti ${ }^{2}$, J. Dissemond', S. Grabbe}

Klinik und Poliklinik für Dermatologie, Venerologie und Allergologie der Universität Essen (Direktor: Prof. Dr. Stephan Grabbe)

Hautklinik der Universität São Paulo, Brasilien (Direktor: Prof. Dr. Evandro A. Rivitti)

\section{Bibliografie}

Dol $10.1055 / \mathrm{s}-2007-966517$

Akt Dermatol 2007; 33:

210-215 @ Georg Thieme

Verlag KG Stuttgart · New York ISSN 0340-2541

Korrespondenzadresse

Dr. Thomas Jansen

Klinik und Poliklinik für

Dermatologie, Venerologie

und Allergologie

der Universität Essen

Hufelandstraße 55

45122 Essen

thomas.jansen@

medizin.uni-essen.de

\section{Zusammenfassung}

$\nabla$

Berichtet wird über die Therapie mit 5\% Kaliumhydroxid in wässriger Lösung bei 21 Kindern im Alter von 2-13 (durchschnittlich 6,4) Jahren mit Molluscum contagiosum. In der Mehrzahl der Fälle waren multiple (durchschnittlich 10) Läsionen vorhanden, die seit 4-8 (durchschnittlich 6,2) Monaten bestanden. Bei 14 Patienten war eine atopische Diathese eruierbar, 3 Patienten hatten ein atopisches Ekzem. Die Lösung wurde von den Eltern zweimal täglich (morgens und abends) aufgetragen, bis es zu einer klinisch erkennbaren Entzündungsreaktion kam. Die Studie wurde von allen Patienten protokollgemäß beendet. Nach 3-6 (durchschnittlich 4) Tagen kam es zu einer Entzündungsreaktion oder oberflächlichen Ulzeration, die nach 14-35 (durchschnitt-

\section{Einleitung}

Das Molluscum contagiosum ist eine benigne, jedoch hochkontagiöse, viral bedingte Hauterkrankung hauptsächlich des Kindesalters, die im Allgemeinen nur eine begrenzte Bestandsdauer aufweist $[2,5,21]$. Daher verbietet sich im Grunde jede Therapie, die nachteilige Folgen, wie z. B. Narben, hinterlässt. Da die Erkrankung häufig, harmlos und selbstlimitierend ist, muss die Behandlung einfach und kostengünstig durchzuführen sein und darf nur wenige Beschwerden verursachen. Andererseits muss sie alle Läsionen verlässlich beseitigen, eine neue Aussaat verhindern und vor Rezidiven schützen.

Angesichts der Vielzahl verschiedener Therapiemöglichkeiten, die mehr oder weniger erfolgreich sind, und der häufig eingesetzten aggressiven physikalischen Behandlungsmethoden, wie z. B. Kürettage oder Kryotherapie, sollte in einer offenen, nicht-kontrollierten Studie, aufbauend auf den ermutigenden Ergebnissen vorangegan- lich 26,7) Tagen zu einer vollständigen Abheilung der Läsionen führte. In einem Nachbeobachtungszeitraum von 2-8 (durchschnittlich 5,1) Monaten traten keine Rezidive auf. Die häufigste Nebenwirkung bei 76,2\% der Patienten bestand in einem kurzen, leichten Brennen am Applikationsort unmittelbar nach Auftragen der Lösung. Zwei Patienten entwickelten eine postinflammatorische Hyperpigmentierung, die sich innerhalb von 3-6 Monaten vollständig zurückbildete. Die Studie bestätigt die gute Wirksamkeit und Verträglichkeit einer 5\%igen Kaliumhydroxid-Lösung in der Behandlung von Molluscum contagiosum im Kindesalter, wobei sich die hohe Compliance der Patienten und ihrer Eltern sowie die einfache Durchführbarkeit der Therapie zu Hause als vorteilhaft erweisen. gener Studien $[6,10,15-17,19]$, die Eignung von $5 \%$ Kaliumhydroxid in wässriger Lösung (Kalilauge) für die Indikation Molluscum contagiosum im Kindesalter geprüft werden.

\section{Material und Methoden \\ $\nabla$}

In die Studie wurden 21 konsekutive Patienten mit Molluscum contagiosum eingeschlossen, die in der Ambulanz der Hautklinik der Universität Essen über einen Zeitraum von 3 Monaten rekrutiert wurden. Patienten mit bekannter Immundefizienz und Vorbehandlung der Mollusken in den vorangegangenen 4 Wochen wurden von der Studie ausgeschlossen. Ebenso wurden Patienten mit Riesenmollusken und Eczema molluscatum ausgeschlossen. Erfasst wurden Alter und Geschlecht, Bestandsdauer der Erkrankung, Lokalisation und Anzahl der Läsionen, Vorhandensein einer atopischen Diathese bzw. Erkrankung aus dem atopischen Formenkreis, Therapieverlauf, 
Tab. 1 Kenndaten der in die Studie eingeschlossenen, mit $5 \%$ iger Kaliumhydroxid-Lösung behandelten Patienten mit Molluscum contagiosum

\begin{tabular}{|c|c|c|c|c|c|c|}
\hline Pat.-Nr. & $\begin{array}{l}\text { Alter (Jahre), } \\
\text { Geschlecht }\end{array}$ & $\begin{array}{l}\text { Lokalisation, } \\
\text { Anzahl }\end{array}$ & $\begin{array}{l}\text { Dauer bis Ent- } \\
\text { zündungsreak- } \\
\text { tion (Tage) }\end{array}$ & $\begin{array}{l}\text { Ergebnis } \\
\text { (Tage) }\end{array}$ & $\begin{array}{l}\text { Nachbeobachtung } \\
\text { (Monate), Rezidiv }\end{array}$ & Nebenwirkungen \\
\hline 1 & $7, w$ & Gesicht, 5 & 4 & Abheilung (28) & 8 , nein & nein \\
\hline 2 & $6, w$ & Stamm, 7 & 3 & Abheilung (14) & 5 , nein & nein \\
\hline 3 & $5, \mathrm{~m}$ & $\begin{array}{l}\text { Stamm, } 10 \\
\text { Arme, } 2\end{array}$ & 5 & Abheilung (21) & 7, nein & nein \\
\hline 4 & $9, w$ & Gesicht, 4 & 4 & Abheilung (28) & 6 , nein & Hyperpigmentierung \\
\hline 5 & $4, w$ & Stamm, 12 & 5 & Abheilung (35) & 4 , nein & nein \\
\hline 6 & $2, m$ & Stamm, 10 & 4 & Abheilung (28) & 4 , nein & nein \\
\hline 7 & $9, \mathrm{~m}$ & $\begin{array}{l}\text { Gesäß, } 4 \\
\text { Beine, } 6\end{array}$ & 6 & Abheilung (35) & 5 , nein & nein \\
\hline 8 & $12, w$ & Stamm, 15 & 4 & Abheilung (21) & 6 , nein & nein \\
\hline 9 & $9, w$ & Arme, 6 & 3 & Abheilung (28) & 8 , nein & nein \\
\hline 10 & $6, w$ & $\begin{array}{l}\text { Gesicht, } 2 \\
\text { Arme, } 3\end{array}$ & 5 & Abheilung (21) & 7, nein & nein \\
\hline 11 & $13, w$ & Gesicht, 4 & 4 & Abheilung (14) & 3 , nein & Hyperpigmentierung \\
\hline 12 & $3, \mathrm{~m}$ & Stamm, 20 & 4 & Abheilung (28) & 4, nein & nein \\
\hline 13 & $2, \mathrm{~m}$ & Stamm, 15 & 4 & Abheilung (35) & 5 , nein & nein \\
\hline 14 & $8, w$ & Beine, 5 & 3 & Abheilung (35) & 4, nein & nein \\
\hline 15 & $7, m$ & Gesicht, 3 & 3 & Abheilung (28) & 2 , nein & nein \\
\hline 16 & $9, w$ & $\begin{array}{l}\text { Gesicht, } 2 \\
\text { Hals, } 1\end{array}$ & 4 & Abheilung (21) & 6 , nein & nein \\
\hline 17 & $6, m$ & Hals, 20 & 4 & Abheilung (14) & 4 , nein & nein \\
\hline 18 & $5, w$ & $\begin{array}{l}\text { Stamm, } 7 \\
\text { Beine, } 3\end{array}$ & 5 & Abheilung (28) & 5 , nein & nein \\
\hline 19 & $2, w$ & $\begin{array}{l}\text { Gesicht, } 2 \\
\text { Stamm, } 14\end{array}$ & 3 & Abheilung (28) & 7, nein & nein \\
\hline 20 & $7, m$ & $\begin{array}{l}\text { Stamm, } 11 \\
\text { Arme, } 6 \\
\text { Beine, } 4\end{array}$ & 4 & Abheilung (35) & 5 , nein & nein \\
\hline 21 & $4, m$ & Stamm, 12 & 4 & Abheilung (35) & 3 , nein & nein \\
\hline
\end{tabular}

Ergebnis, Nachbeobachtungszeitraum und Rezidivhäufigkeit sowie Nebenwirkungen ( Tab. 1). Von den Eltern wurde die Einwilligung zur Teilnahme an der Studie eingeholt.

Zur Anwendung kam Kaliumhydroxid in 5\%iger wässriger Lösung. Die Eltern der betroffenen Kinder wurden instruiert, die Lösung mit einem Kunststoffspatel auf sämtliche Läsionen zu Hause aufzutragen. Die Anwendung wurde zweimal täglich (morgens und abends) wiederholt. Sie war zu beenden, sobald eine klinisch erkennbare Entzündungsreaktion die Abheilung der Läsionen anzeigte. Bei Auftreten neuer Läsionen während der Studiendauer wurden diese ebenfalls behandelt und die Daten erfasst.

Die Beurteilung des Status erfolgte jeweils durch ein und denselben Untersucher an den Tagen 0, 14, 21, 28 und 35. Nach Registrierung der klinischen Abheilung schloss sich ein mehrmonatiger Nachbeobachtungszeitraum zur Verlaufskontrolle von bei Therapieende nachweisbaren Nebenwirkungen und zur Ermittlung der Rezidivhäufigkeit an.

\section{Ergebnisse}

$\nabla$

In die Studie wurden 21 kaukasische Patienten mit Hauttyp I und II nach Fitzpatrick, 9 Jungen und 12 Mädchen im Alter von 2-13 (durchschnittlich 6,4) Jahren, eingeschlossen. Die Anzahl der Läsionen variierte mit 1-20 erheblich, wobei bei der überwiegenden Mehrzahl der Patienten multiple Läsionen (durchschnittlich 10) vorhanden waren. Die meisten Läsionen waren am Stamm (61,9\%) lokalisiert, nachfolgend fanden sie sich auch an den Extremitäten (16,3\%), im Gesicht (10,2\%), am Hals (9,8\%) und am Gesäß (1,9\%). Die Bestandsdauer der Erkrankung lag bei 4-8 (durchschnittlich 6,2) Monaten.

Eine atopische Diathese war bei 14 Patienten (66,7\%) eruierbar, 3 Patienten (14,3\%) hatten ein gering ausgeprägtes atopisches Ekzem.

Sämtliche Patienten beendeten die Studie. Bei ihnen trat nach 3-6 (durchschnittlich 4) Tagen eine klinisch erkennbare Entzündungsreaktion oder oberflächliche Ulzeration ein ( $\bullet$ Abb. 1). Eine vollständige Abheilung war nach 14-35 (durchschnittlich 26,7) Tagen zu verzeichnen.

Bei keinem der Patienten wurden Rezidive während des Nachbeobachtungszeitraums von 2-8 (durchschnittlich 5,1) Monaten beobachtet.

Die meisten Patienten (76,2\%) gaben ein kurzes, etwa 1 - 2 min anhaltendes, leichtes, gut erträgliches Brennen am Applikationsort an, das oftmals nur nach zunehmender Applikationsdauer entstand. Schwerwiegendere Nebenwirkungen wurden bei $2 \mathrm{~Pa}-$ tienten (9,5\%) mit Lokalisation der Läsionen im Gesicht beobachtet und umfassten postinflammatorische Hyperpigmentierungen am Applikationsort, die sich innerhalb des Nachbeobachtungszeitraums innerhalb von 3-6 Monaten vollständig zurückbildeten. Bei keinem der 3 Patienten mit atopischem Ekzem kam es unter der Therapie zu einer nennenswerten Verschlechterung des Ekzems. Bei keinem der Patienten blieben nach der Abheilung der Läsionen Narben zurück. 


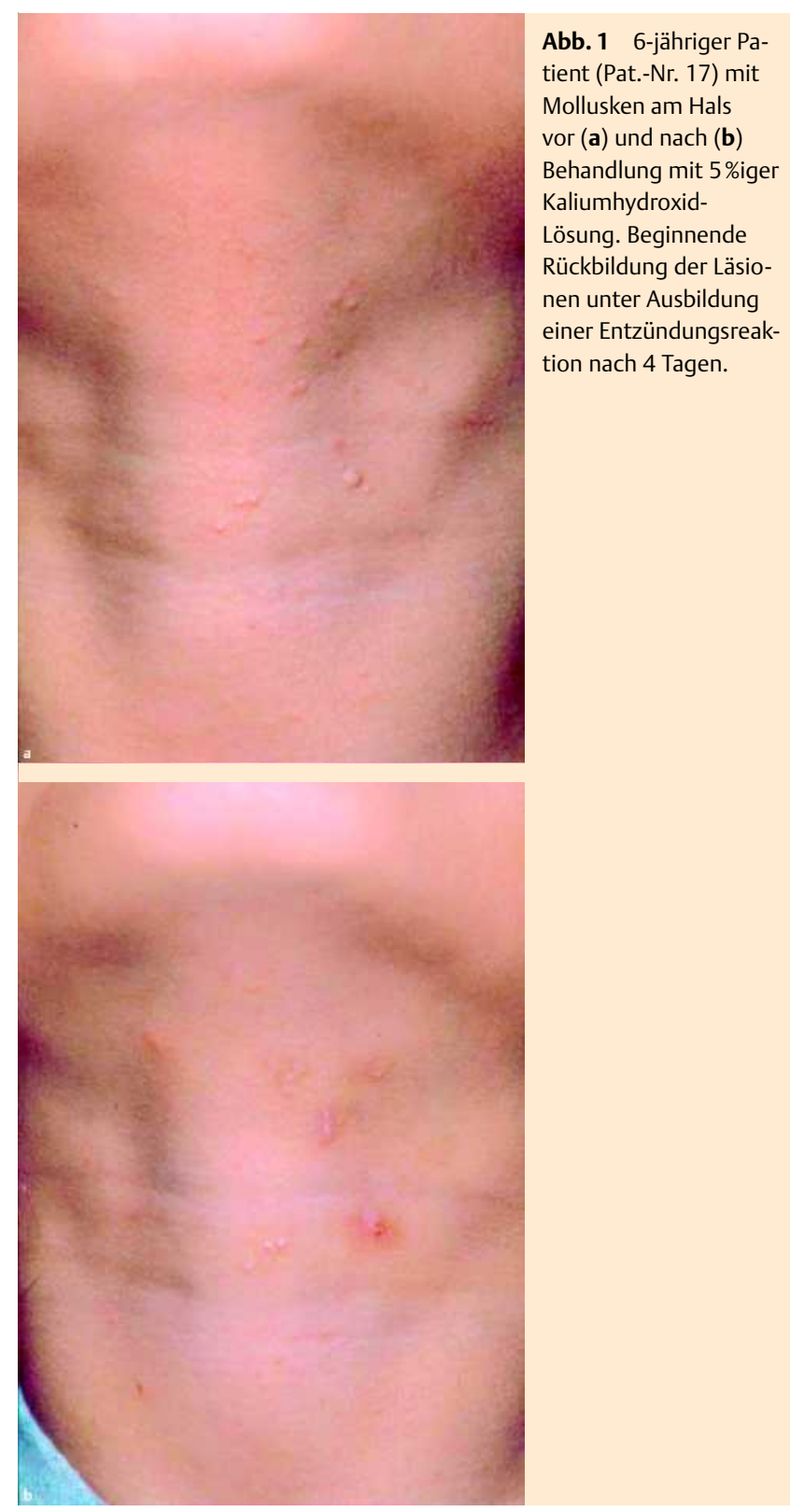

\section{Diskussion}

$\nabla$

Das Molluscum-contagiosum-Virus (MCV) ist das einzige Mitglied des Genus Molluscipoxvirus [2,5,21]. Es verursacht neben dem Variolavirus, dem Erreger der Pocken, die einzige noch verbliebene humanspezifische Pockenviruserkrankung. Es handelt sich um ein großes $(200-300 \mathrm{~nm})$, quaderförmiges, doppelsträngiges DNS-Virus, das sich im Zytoplasma der infizierten epithelialen Zellen vermehrt. Es gibt mindestens 4 Genotypen (MCV 1-4), die sich hinsichtlich Morphologie und Topografie der Läsionen nicht unterscheiden. Das MCV ist weltweit verbreitet, wobei der Erreger direkt durch Schmierinfektion von Mensch zu Mensch (Auto- und Heteroinokulation), aber auch indirekt durch infizierte Gegenstände übertragen wird. Die Inkubationszeit liegt bei 2 Wochen bis 6 Monaten.

Die Erkrankung (Molluscum oder Epithelioma contagiosum) ist auf die Haut und Schleimhaut beschränkt und manifestiert sich in Form kleiner (Durchmesser 1-10 mm), hautfarbener oder weißlicher, derber Papeln, die typischerweise eine zentrale Ein- senkung aufweisen, daher stammt auch die Bezeichnung Dellwarzen. Die benignen Neubildungen enthalten ein wachsartiges Material, das sich aus Zelldebris und zahlreichen Pockenviruspartikeln zusammensetzt. Die klinische Diagnose lässt sich mikroskopisch aus dem Exprimat einer Läsion sichern, wobei die Giemsa-Färbung eines Ausstrichpräparates große Einschlusskörperchen (Molluskumkörperchen) in zerfallenden Keratinozyten zeigt. Mollusken finden sich gewöhnlich gruppiert in ein oder zwei Hautregionen, kommen aber auch locker disseminiert vor. Während die meisten Patienten weniger als 20 Läsionen aufweisen, können sich bei einigen mehrere Hundert entwickeln. Die Läsionen sind in der Regel symptomlos, gelegentlich bedingen sie jedoch Juckreiz, und in ihrer Umgebung kann sich eine ekzemartige Reaktion entwickeln. Grundsätzlich handelt es sich um eine selbstlimitierende Erkrankung mit Neigung zu spontaner Involution innerhalb von 6-9 Monaten, jedoch können die Läsionen auch jahrelang persistieren. Die Abheilung erfolgt in der Regel ohne Narben.

In der überwiegenden Mehrzahl der Fälle sind Kinder im Schulalter betroffen $[2,5,21]$. Das gilt besonders für tropische Regionen, in denen bis zu 10\% der Schulkinder Mollusken aufweisen, wobei enger körperlicher Kontakt, luftige Bekleidung und schlechte körperliche Hygiene als begünstigende Faktoren anzusehen sind. In kühleren und besser entwickelten Ländern tritt die Erkrankung auch in einem höheren Lebensalter auf, wobei eine Häufung bei Kontaktsportlern und Besuchern von öffentlichen Badeanstalten gesehen wird. Im Kindesalter ist eine Bevorzugung spezieller Körperregionen nicht erkennbar, sondern die Mollusken befallen das gesamte Integument und die angrenzenden Schleimhäute, sparen aber gewöhnlich die behaarte Kopfhaut, Handflächen und Fußsohlen aus. In dieser Altersgruppe ist auch selten ein genitaler Befall möglich, ohne dass dies zwangsläufig als Hinweis auf sexuellen Missbrauch zu werten ist. Mollusken sind hochkontagiös, besonders bei Kindern, und können daher leicht zwischen Geschwistern oder im Kindergarten übertragen werden.

Ein weiterer Häufigkeitsgipfel findet sich durch venerische Kontaktinfektion bei sexuell-aktiven Erwachsenen, wobei bevorzugt der Genitalbereich und die angrenzende Haut der Oberschenkel und des unteren Abdomens befallen sind [14]. Eine starke Häufung ist bei HIV-Infektion und anderen angeborenen und erworbenen, auch iatrogenen, Immundefizienzen zu verzeichnen. Bei diesen Patienten fällt nicht nur die Anzahl der Läsionen, sondern auch das Auftreten aberranter gigantischer, großknotiger Konglomerate (Mollusca contagiosa gigantea, Riesenmollusken) mit einem Durchmesser von $3 \mathrm{~cm}$ oder mehr auf. Bei HIV-Infizierten gibt es eine inverse Beziehung zwischen CD4-Zellzahlen und der Anzahl der Läsionen, daher gelten Mollusken als eine Markererkrankung der fortgeschrittenen HIV-Infektion [8]. In dieser Patientengruppe finden sich die Läsionen nicht nur im Genitalbereich und in den angrenzenden Hautpartien, sondern auch am oberen Rumpfabschnitt, Hals, an der behaarten Kopfhaut und besonders auch im Gesicht. Insgesamt wird die Häufigkeit von Mollusken bei HIV-Infizierten auf 5-18\% geschätzt [2]. Eine erhöhte Infektionsbereitschaft ist auch bei eingeschränkter epidermaler Barrierefunktion, wie sie für das atopische Ekzem kennzeichnend ist, gegeben.

Obwohl das Molluscum contagiosum eine benigne Veränderung darstellt und spontan abheilen kann, erfordert die mögliche Übertragung auf andere Kinder und die Ausbreitung der Läsionen am betroffenen Kind oftmals eine therapeutische Intervention [11]. Trotz zahlreicher Behandlungsmöglichkeiten gibt es 
bis heute keine spezifische antivirale Therapie des Molluscum contagiosum. Als Therapie der Wahl gilt die Abtragung der Mollusken mit einer Kürette (scharfer Löffel, Ringkürette) oder gebogenen (Eihaut-)Pinzette [18]. Schwierigkeiten ergeben sich bei starker Mollusken-Dissemination und ungünstiger Lokalisation (z.B. Gesicht, Hals oder Genitalregion). Durch perkutane Anästhesie mit einer Lidocain-Prilocain-Creme $\left(\mathrm{EMLA}^{\circledR}\right)$ [18], unter Okklusion 30-60 min vorher aufgetragen, ist eine schmerzfreie Entfernung zwar auch dann noch gelegentlich möglich, jedoch lässt sich in vielen Fällen, besonders bei unruhigen Kindern, ein operativer Eingriff in Sedierung oder Allgemeinnarkose nicht umgehen. Eine Kryotherapie mit flüssigem Stickstoff, der entweder direkt unter Verwendung einer Schablone oder indirekt durch Anwendung eines Stempelaufsatzes auf die Läsion einwirkt, ist zwar in der Regel weniger schmerzhaft, jedoch zeitaufwendiger als die Abtragung mit Kürette oder Pinzette, außerdem stellt sich der Behandlungserfolg selten nach einmaliger Applikation ein, sodass mehrere Sitzungen erforderlich sind [1]. Die gelegentlich eingesetzten gepulsten $\mathrm{CO}_{2}$ - und Farbstoff-Laser [7] sowie Elektrokauter lassen hinsichtlich Praktikabilität und Verträglichkeit keinen Vorteil erkennen.

Bei den empfohlenen konservativen Therapieverfahren gibt es zahlreiche Alternativen, die jede für sich ein hohes Maß an Kooperation und Zuverlässigkeit und auch Geduld der behandelnden Eltern voraussetzen. Salizylsäure als Pflaster (Guttaplast ${ }^{\circledR}$ ) [13] oder Vitamin-A-Säure (Tretinoin) [14] werden gelegentlich mit Erfolg eingesetzt, können jedoch zu einer erheblichen Irritation der periläsionalen Haut führen. Die topische Applikation des Immunmodulators Imiquimod (Aldara ${ }^{\circledR}$ ) scheint wirksam zu sein [9], wobei bislang keine Zulassung für die Behandlung von Kindern vorliegt, die erforderliche Behandlungsdauer nicht ausreichend untersucht ist und die Behandlungskosten erheblich sind. Weitere konservative Therapiemaßnahmen, die mit variablen Ergebnissen eingesetzt werden, umfassen die topische Applikation von Podophyllotoxin [1], Cantharidin [20], Trichloressigsäure, Phenol, Silbernitrat [12] oder Jod [13]. Es ist anzunehmen, dass einige der eingesetzen chemischen Substanzen durch epidermale Schädigung und dadurch bedingte Induktion einer Immunantwort zur Viruselimination führen. Erfolge wurden schließlich auch mit oraler Cimetidin-Therapie berichtet, wobei immunmodulierende Eigenschaften der Substanz (Hemmung der Suppressor-Lymphozyten-Funktion und Verbesserung der T-Zell-Immunität) vermutet wurden [3].

Kaliumhydroxid wird in 10\%iger Konzentration als Lösungsmittel für mikroskopische Nativpräparate verwendet, um Pilzelemente nachzuweisen. Nagelsubstanz und Epidermisstücke, die keratinreich sind, fallen unter der Laugeneinwirkung zu einer dünnen, transparenten Schicht zusammen, die Myzel in Einzelheiten erkennen lässt. In hoher Konzentration dringt Kaliumhydroxid tief in die Haut ein und zerstört sie, in niedriger Konzentration führt es zu einer irritativen Dermatitis [4]. Es gibt inzwischen gute Belege dafür, dass sich Kaliumhydroxid auch zur Behandlung von Molluscum contagiosum im Kindesalter eignet. Romiti und Mitarbeiter [16] aus Brasilien beschrieben die topische Applikation einer 10\%igen Kaliumhydroxid-Lösung, wie sie auch zur Anfertigung von mykologischen Nativpräparaten verwendet wird, zur Behandlung von Kindern mit Molluscum contagiosum. Das Protokoll entsprach einer offenen, nicht-kontrollierten Studie. Berichtet wurde von 35 Kindern im Alter von 7 Monaten bis 15 (durchschnittlich 6) Jahren, die Läsionen vorwiegend im Gesicht, am Stamm und/oder den Extremitäten aufwiesen. Patienten mit bekannter Immundefizienz oder periorbi- talen Läsionen wurden von der Studie ausgeschlossen. 15 (42,9\%) der Patienten hatten eine atopische Diathese. Bei allen Patienten kam es bei zweimal täglicher Anwendung nach durchschnittlich 5-7 Tagen zu einer klinisch erkennbaren Entzündungsreaktion oder oberflächlichen Ulzeration, welche den zeitlichen Endpunkt der topischen Applikation markierte. Drei Patienten brachen die Therapie wegen starken Brennens am Applikationsort $(n=2)$ und bakterieller Superinfektion $(n=1)$ vorzeitig ab. Bei 91,4\% der Patienten kam es innerhalb von durchschnittlich 30 Tagen zu einer vollständigen Abheilung der Läsionen, bei zugleich überschaubaren Nebenwirkungen, vornehmlich einem kurzen Brennen (Dauer 1-2 min) unmittelbar nach Applikation der Lösung bei den meisten Patienten. Bei 9 (28,1\%) der Patienten, die 10\%ige Kaliumhydroxid-Lösung anwendeten, traten schwerwiegendere Nebenwirkungen auf, darunter eine zumeist temporäre Hyper- und/oder Hypopigmentierung bei 8 (25\%) der Patienten. Bei einem Patienten trat trotz korrekter Anwendung eine hypertrophe Narbe an einer der behandelten Stellen auf, die sich innerhalb von 6 Monaten nach Therapieende zu einer wenig auffälligen Narbe zurückbildete. Die Autoren hoben die Zufriedenheit der Eltern mit dieser einfachen, zu Hause durchzuführenden Therapie hervor.

In einer kurzen Mitteilung berichtete dieselbe Arbeitsgruppe darüber hinaus, dass mit einer 5\%igen Kaliumhydroxid-Lösung, ebenfalls zweimal täglich aufgetragen, bei 20 Kindern mit Molluscum contagiosum vergleichbare Heilungsraten (innerhalb von durchschnittlich 6 Wochen) bei zugleich deutlich reduziertem Nebenwirkungsprofil erzielt wurden [17]. Das Brennen am Applikationsort der Lösung blieb aus oder war gering, Pigmentveränderungen kamen nicht vor. Sogar perigenitale und perianale Läsionen ließen sich auf diese Weise behandeln, ohne dass starke Irritationen auftraten.

Eine andere Studie aus Indien berichtete über die Anwendung einer 20\%igen Kaliumhydroxid-Lösung, einmal täglich (abends) aufgetragen, bei 27 Kindern im Alter von 8 Monaten bis 14 (durchschnittlich 5,8) Jahren mit Molluscum contagiosum [10]. Bei der überwiegenden Mehrzahl (74,1\%) der Kinder war ein disseminierter Befall mit durchschnittlich 13 Läsionen vorhanden. Drei Patienten brachen die Studie wegen lokaler Unverträglichkeit $(n=2)$ oder bakterieller Superinfektion $(n=1)$ vorzeitig ab. Bei den übrigen 24 (88,9\%) Patienten trat nach durchschnittlich 5-7 Tagen eine Entzündungsreaktion oder oberflächliche Ulzeration und nach 13-22 (durchschnittlich 17) Tagen eine vollständige Abheilung der Läsionen ein. Die meisten Kinder gaben ein kurzes Brennen unmittelbar nach dem Auftragen der Lösung an. Bei 8 (29,6\%) der Patienten wurden schwerwiegendere Nebenwirkungen beobachtet, darunter Hypopigmentierung $(n=4)$, starkes Brennen am Applikationsort $(\mathrm{n}=2)$ und bakterielle Superinfektion $(n=2)$.

Eine weitere, Plazebo-kontrollierte Studie aus Peru untersuchte den Effekt von 15\%iger Kaliumhydroxid-Lösung, einmal täglich (abends) aufgetragen, bei 46 Patienten unterschiedlichen Alters (zumeist < 5 Jahre) mit Molluscum contagiosum [6]. Die Altersstruktur, Verteilung der Läsionen (in 83,3\% Befall des Stammes) und Erkrankungsdauer vor Therapiebeginn (4,9 Monate) in der Verum- und Plazebo-Gruppe waren vergleichbar, allerdings waren in der Verum-Gruppe mehr Mädchen (3,6:1 vs. 1:1) und mehr Patienten mit atopischem Ekzem (28,57\% vs. $10 \%)$ vorhanden. Nach Beendigung der Therapie mit 15\%iger Kaliumhydroxid-Lösung waren bei $57 \%$ der Patienten die Läsionen abgeheilt, bei 35,7\% eine mittlere Besserung und bei 7,14\% eine leichte Besserung eingetreten. Dagegen war in der Plazebo-Gruppe bei $30 \%$ 
ein Heilungserfolg zu verzeichnen, bei 30\% kam es zu einer mittleren und bei $20 \%$ zu einer leichten Besserung, bei $10 \%$ änderte sich der Zustand nicht, und bei weiteren $10 \%$ trat sogar eine Verschlechterung ein. Bei keinem der Patienten, die Plazebo erhielten, jedoch bei $21,4 \%$ der Patienten, die mit $15 \%$ iger Kaliumhydroxid-Lösung behandelt wurden, trat die Abheilung vor Ablauf von 30 Tagen ein. In der Verum-Gruppe trat bei 92,85\% der Patienten als häufigste Nebenwirkung ein Brennen am Applikationsort auf, gefolgt von Hyper- (71,42\%) und Hypopigmentierung (50\%), Ulzeration (28,57\%), Narbenbildung (14,25\%) und Schmerzen (7,14\%). Wenngleich hinsichtlich der Wirksamkeit, vermutlich wegen der geringen Stichprobengröße, kein statistisch signifikanter Unterschied zum Plazebo festgestellt werden konnte, erwies sich die Therapie mit 15\%iger KaliumhydroxidLösung als wirksam, sicher und einfach in der Anwendung.

Short und Mitarbeiter [19] aus Großbritannien legten kürzlich die erste doppelblinde, Plazebo-kontrollierte Studie zur Anwendung von 10\%iger Kaliumhydroxid-Lösung, zweimal täglich aufgetragen, bei 20 Kindern im Alter von 2-12 Jahren mit Molluscum contagiosum vor. 19 (95\%) der Patienten beendeten die Studie. In der Verum-Gruppe heilten die Läsionen bei 7 (70\%) der Patienten nach durchschnittlich 54 Tagen vollständig ab, dagegen zeigten 2 (20\%) der Patienten keine Besserung. Schwerwiegendere Nebenwirkungen traten bei 4 Patienten auf, darunter starkes Brennen am Applikationsort $(n=2)$, das bei einem Patienten zum Therapieabbruch führte, und temporäre Hyperpigmentierung $(n=2)$. Beide Kinder, die eine Pigmentstörung entwickelten, hatten einen hellen Hauttyp (Typ II nach Fitzpatrick). In der Plazebo-Gruppe trat bei $2(20 \%)$ der Patienten eine vollständige Abheilung ein, 8 (80\%) zeigten keine Besserung. Die Überlegenheit der 10\%igen Kaliumhydroxid-Lösung gegenüber dem Plazebo (77\% vs. $20 \%$ Abheilung) war zwar offensichtlich, jedoch ließ sich zwischen Verum- und Plazebo-Gruppe aufgrund der geringen Stichprobengröße kein statistisch signifikanter Unterschied berechnen.

Aufbauend auf den positiven Erfahrungen früherer Untersuchungen führten wir eine Therapie mit $5 \%$ iger KaliumhydroxidLösung bei 21 Kindern im Alter von 2-13 (durchschnittlich 6,4) Jahren mit Molluscum contagiosum durch. Die Wahl der Konzentration fiel auf 5\%, da Romiti und Mitarbeiter [17] über vergleichbare Therapieerfolge bei günstigerem Nebenwirkungsspektrum durch Halbierung der ursprünglich eingesetzten Konzentration berichtet hatten. In der Mehrzahl der Fälle waren multiple (durchschnittlich 10) Läsionen überwiegend am Stamm vorhanden, die seit 4-8 (durchschnittlich 6,2) Monaten bestanden. Bei 14 Patienten war eine atopische Diathese eruierbar, 3 Patienten hatten ein atopisches Ekzem.

Die Lösung wurde von den Eltern zweimal täglich (morgens und abends) zu Hause aufgetragen, bis es zu einer klinisch erkennbaren Entzündungsreaktion kam. Die Studie wurde von allen Patienten beendet. Nach 3-6 (durchschnittlich 4) Tagen kam es zu einer Entzündungsreaktion oder oberflächlichen Ulzeration, die nach 14 - 35 (durchschnittlich 26,7) Tagen zu einer vollständigen Abheilung der Läsionen führte. In dieser Studie ergab sich, vermutlich durch individuelle Unterschiede (z.B. Hauttypen) bedingt, eine deutlich kürzere durchschnittliche Dauer bis zum Einsetzen der vollständigen Abheilung als von Romiti und Mitarbeitern [17] mitgeteilt (durchschnittlich 6 Wochen).

Die häufigste Nebenwirkung bei 76,2\% der Patienten bestand, analog zu den Mitteilungen von Romiti und Mitarbeitern [17], in einem leichten Brennen über 1-2 min am Applikationsort unmittelbar nach Auftragen der Lösung. Zwei Patienten entwi- ckelten eine postinflammatorische Hyperpigmentierung, die sich innerhalb von 3-6 Monaten vollständig zurückbildete. Das Nebenwirkungsspektrum bei $5 \%$ iger Konzentration ließ erhebliche Vorteile gegenüber den zuvor eingesetzten KaliumhydroxidKonzentrationen von $10 \%$ [16,19], 15\% [6] und 20\% [10] erkennen. Insbesondere wurden weder starkes Brennen noch Schmerzen, bakterielle Superinfektionen, Narbenbildungen oder persistierende Pigmentstörungen beobachtet. Hervorzuheben ist, dass sich die $5 \%$ ige Kaliumhydroxid-Therapie auch bei Patienten mit atopischer Diathese komplikationslos durchführen ließ.

Selbstverständlich kann eine spontane Involution in der vorliegenden Studie und auch in den vorangegangenen Studien nicht sicher ausgeschlossen werden, jedoch lässt das rasche Einsetzen einer vollständigen Abheilung (nach durchschnittlich 26,7 Tagen) bei mehrmonatiger (durchschnittlich 5,1-monatiger) Rezidivfreiheit einen klaren Vorteil der eingesetzten 5\%igen Kaliumhydroxid-Lösung im Vergleich zu den üblichen Spontanverläufen erkennen. Weiterhin trug die Konsequenz und Sorgfalt bei der Durchführung der Anwendung, wie sie im Rahmen einer derartigen Studie unter ärztlicher Kontrolle gewährleistet war, zum Therapieerfolg bei.

In Zusammenschau der bisher vorliegenden Daten stellt 5\%ige Kaliumhydroxid-Lösung eine kostengünstige, wirksame, nebenwirkungsarme, nicht-invasive Therapieoption bei Molluscum contagiosum im Kindesalter dar. Der Wirkstoff steht mittlerweile als Medizinprodukt (InfectoDell ${ }^{\circledR}$ ) zur Verfügung. Vorteilhaft wirkt sich die hohe Compliance der behandelten Kinder bzw. deren Eltern und die einfache Durchführbarkeit der Therapie zu Hause aus. Die Lösung ist zweimal täglich bis zu einer klinisch erkennbaren Entzündungsreaktion aufzutragen. Erfahrungen bei Patienten mit angeborener oder erworbener Immundefizienz liegen bisher nicht vor. Die Überlegenheit der Therapie des Molluscum contagiosum mit 5\%iger Kaliumhydroxid-Lösung sollte künftig in Plazebo-kontrollierten Studien an großen Kollektiven gesichert werden. In weiteren Studien ist zu prüfen, ob Kaliumhydroxid-Lösung auch in niedrigeren Konzentrationen als bisher (z. B. 2,5\%) zu vergleichbaren Abheilungsquoten bei noch weiter optimiertem Nebenwirkungsprofil führt.

\section{Abstract}

\section{Evaluation of the Efficacy and Tolerability \\ of $5 \%$ Potassium Hydroxide Solution in the Treatment of Molluscum Contagiosum in Childhood $\nabla$}

In a clinical study, 21 children aged $2-13$ (mean 6.4 ) years with molluscum contagiosum were evaluated for efficacy and tolerability of treatment with topical $5 \%$ potassium hydroxide aqueous solution. Most patients had multiple (mean 10) lesions which were present for 4-8 (mean 6.2) months. A history of atopy was noted in 14 patients, while 3 patients had atopic eczema. The solution was applied by the parents of affected children, twice daily (at morning and evening), on each lesion, until all lesions underwent clinically visible inflammatory reaction. All patients completed the study. Time until development of inflammation or superficial ulceration was 3-6 (mean 4) days, leading to complete resolution of lesions after 14-35 (mean 26.7) days. In a follow-up period of $2-8$ (mean 5.12) months, no recurrences were seen. Most common side-effect observed in $76.2 \%$ of patients was transient minor stinging immediately after topical application. Two patients developed postinflamma- 
tory hyperpigmentation, which resolved completely within 3-6 months. The study confirms the efficacy and good tolerability of $5 \%$ potassium hydroxide solution in the treatment of molluscum contagiosum in childhood, with the advantage of high compliance of patients and their parents as well as easy practicability when performed as home treatment.

\section{Literatur}

1 Barton SE, Chard S. Facial molluscum: treatment with cryotherapy and podophyllotoxin. Int J STD AIDS 2002; 13: 277-278

2 Brown J, Janniger CK, Schwartz RA, Silverberg NB. Childhood molluscum contagiosum. Int J Dermatol 2006; 45: 93 - 99

3 Dohil M, Prendiville JS. Treatment of molluscum contagiosum with oral cimetidine: clinical experience in 13 patients. Pediatr Dermatol 1996; 13: $310-312$

4 Frosch PJ. Cutaneous irritation. In: Rycroft RH, Menné T, Frosch PJ (eds). Textbook of Contact Dermatitis. 2nd edn. Berlin: Springer, 1995: $311-354$

5 Gottlieb SL, Myskowsky PL. Molluscum contagiosum. Int J Dermatol 1994; 33: $453-461$

6 Hinostroza-Da-Conceicao D, Beirana-Palencia A. Tratamento del mollusco contagioso con hidróxido de potasio al $15 \%$ en solución acquosa. Dermatol Peru 2004; 14: 185 - 191

7 Hughes PS. Treatment of molluscum contagiosum with the 585-nm pulsed dye laser. Dermatol Surg 1998; 24: 229-230

8 Husak R, Garbe C, Orfanos CE. Mollusca contagiosa bei HIV-Infektion. Klinische Manifestation, Beziehung zum Immunstatus und prognostische Wertigkeit bei 39 Patienten. Hautarzt 1998; 48: 103-109

9 Liota E, Smith KJ, Buckley R, Menon P, Skelton H. Imiquimod therapy for molluscum contagiosum. J Cutan Med Surg 2000; 4: 76-82
10 Mahajan BB, Gupta RR. Topical 20\% $\mathrm{KOH}$ - An effective therapeutic modality for molluscum contagiosum in children. Ind J Dermatol Venereol Leprol 2003; 69: 175-177

11 Myskowsky PL. Molluscum contagiosum. New insights, new directions. Arch Dermatol 1997; 133: 1039-1041

12 Nizeki K, Hashimoto $K$. Treatment of molluscum contagiosum with silver nitrate paste. Pediatr Dermatol 1999; 16: 395-397

13 Ohkuma M. Molluscum contagiosum treated with iodine solution and salicylic acid plaster. Int J Dermatol 1990; 29: 443-445

14 Papa CM, Berger RS. Venereal herpes-like molluscum contagiosum: treatment with tretinoin. Cutis 1976; 18: 537-540

15 Romiti $R$. Kaliumhydroxid als neue Therapieoption bei Mollusca contagiosa. In: Jansen T, Abeck D, Grabbe S (Hrsg). Aktuelle Trends in der pädiatrischen Dermatologie, Bd 2. Berlin: Grosse, 2006: 148 - 150

16 Romiti R, Ribeiro AP, Grinblat BM, Rivitti EA, Romoti N. Treatment of molluscum contagiosum with potassium hydroxide: a clinical approach in 35 children. Pediatr Dermatol 1999; 6: 228-231

17 Romiti R, Ribeiro AP, Romiti N. Evaluation of the effectiveness of $5 \%$ potassium hydroxide for the treatment of molluscum contagiosum. Pediatr Dermatol 2000; 17: 495

18 Rosdahl I, Edmar B, Gisslen H et al. Curretage of molluscum contagiosum in children: analgesia by topical application of a lidocaine/prilocaine cream (EMLA). Acta Derm Venereol (Stockh) 1988; 68: 149-153

19 Short KA, Fuller LC, Higgins EM. Double-blind, randomized, placebocontrolled trial of the use of topical $10 \%$ potassium hydroxide solution in the treatment of molluscum contagiosum. Pediatr Dermatol 2006; 23: $279-281$

20 Silverberg NB, Sidbury R, Mancini AJ. Childhood molluscum contagiosum: experience with cantharidin therapy in 300 patients. J Am Acad Dermatol 2000; 43: 503-507

21 Smith KJ, Yeager J, Skelton H. Molluscum contagiosum: its clinical, histopathologic, and immunohistochemical spectrum. Int J Dermatol 1999; 38: 664-672 